\title{
Analytic theory of fiber-optic Raman polarizers
}

\author{
V. V. Kozlov, ${ }^{* 1,2}$ J. Nuño, ${ }^{3}$ J. D. Ania-Castañón, ${ }^{3}$ and S. Wabnitz ${ }^{1}$ \\ ${ }^{1}$ Department of Information Engineering, Università di Brescia, Via Branze 38, 25123 \\ Brescia, Italy \\ ${ }^{2}$ Department of Physics, St.-Petersburg State University, Petrodvoretz, St.-Petersburg, 198504, \\ Russia \\ ${ }^{3}$ Instituto de Optica, IO-CSIC, Consejo Superior de Investigaciones Cientficas, Serrano 121, \\ 28006 Madrid, Spain \\ *victor.kozlov@email.com
}

\begin{abstract}
The Raman polarizer is a Raman amplifier which not only amplifies but also re-polarizes light. We propose a relatively simple and analytically tractable model - the ideal Raman polarizer, for describing the operation of this device. The model efficiently determines key device parameters such as the degree of polarization, the alignment parameter, the gain and the RIN variance.
\end{abstract}

(C) 2012 Optical Society of America

OCIS codes: (190.5650) Raman effect; (060.2320) Fiber optics amplifiers and oscillators; (190.4370) Nonlinear optics, fibers.

\section{References and links}

1. M. Martinelli, M. Cirigliano, M. Ferrario, L. Marazzi, and P. Martelli, "Evidence of Raman-induced polarization pulling," Opt. Express 17, 947-955 (2009).

2. V. V. Kozlov, J. Nuño, J. D. Ania-Castañón, and S, Wabnitz, “Theory of fiber optic Raman polarizers,” Opt. Lett. 35, 3970-3972 (2010).

3. V. V. Kozlov, J. Nuño, J. D. Ania-Castañón, and S. Wabnitz, "Theoretical study of optical fiber Raman polarizers with counterpropagating beams," J. Lightwave Techn. 29, 341-347 (2011).

4. L. Ursini, M. Santagiustina, and L. Palmieri, "Raman nonlinear polarization pulling in the pump depleted regime in randomly birefringent fibers,” IEEE Photon. Techn. Lett. 23, 254-256 (2011).

5. N. J. Muga, M. F. S. Ferreira, and A. N. Pinto, "Broadband polarization pulling using Raman amplification," Opt. Express 19, 18707-18712 (2011).

6. P. Morin, S. Pitois, and J. Fatome, "Simultaneous polarization attraction and Raman amplification of a light beam in optical fibers," J. Opt. Soc. Am. B 29, 2046-2052 (2012).

7. M. Ferrario, V. Gilardone, P. Martelli, L. Marazzi, and M. Martinelli, "Effective All-Optical Polarization Control Induced by Raman Nonlinear Amplification," in 36th European Conference on Optical Communications (IEEE, 2010), paper P1.19.

8. F. Chiarello, L. Ursini, L. Palmieri, and M. Santagiustina, "Polarization attraction in counterpropagating fiber Raman amplifiers," IEEE Photon. Techn. Lett. 23, 1457-1459 (2011).

9. S. Sergeyev and S. Popov, "Two-section fiber optic Raman polarizer," IEEE J. Quantum Electron 48, 5660 (2012).

10. V. V. Kozlov and S. Wabnitz, "Suppression of relative intensity noise in fiber-optic Raman polarizers, IEEE Photon. Techn. Lett., 23, 1088-1090 (2011).

11. V. V. Kozlov, J. Nuño, J. D. Ania-Castañón, and S. Wabnitz, "Multichannel Raman polarizer with suppressed relative intensity noise for wavelength division multiplexing transmission lines," Opt. Lett. 37, 2073-2075 (2012).

12. V. V. Kozlov and S. Wabnitz, "Silicon Raman polarizer," Opt. Lett. 37, 737739 (2012).

13. S. V. Sergeyev, "Activated polarization pulling and de-correlation of signal and pump states of polarization in a fiber Raman amplifier," Opt. Express 19, 24268-24279 (2011).

14. Q. Lin and G. P. Agrawal, "Vector theory of stimulated Raman scattering and its application 6to fiber-based Raman amplifiers," J. Opt. Soc. Am. B 20, 1616-1631 (2003).

\#176299 - \$15.00 USD Received 14 Sep 2012; revised 17 Oct 2012; accepted 17 Oct 2012; published 19 Nov 2012

(C) 2012 OSA

19 November 2012 / Vol. 20, No. 24 / OPTICS EXPRESS 27242 


\section{Introduction}

Raman-based polarization attraction belongs to a broad class of effects related to light-by-light control in optical waveguides. Models for such control are essentially nonlinear and usually imply the use of a high-intensity beam to modify the properties of the medium (for instance its refractive index or absorption coefficient) such that propagation of a weaker probe beam through the nonlinearly modified medium is affected in a substantial and controllable way. The Raman polarizer was first experimentally demonstrated and numerically validated in a work by Martinelli et.al. [1]. The authors demonstrated that initially unpolarized light is amplified and simultaneously re-polarized in the course of propagation through a low-PMD randomly birefringent telecom fiber (here PMD stands for polarization mode dispersion). Namely, the state of polarization (SOP) of the signal beam at the output of the fiber is attracted towards the SOP of the outcoming pump beam. Soon after this pioneering paper, quite a number of contributions appeared aimed at the theoretical description of the operation of Raman polarizers in both coand counter-propagating configurations, as well as at the study of potential applications such as multi-channel repolarization, enhanced amplification or silicon-based polarizers , [2-13]. All of these papers provided purely numerical, albeit rigorous, studies. It would, still, be certainly desirable to have access to a simple and analytically tractable model, capable of describing the main features of Raman-based polarizers. Here we report such a model, valid for the characterization of Raman polarizers both in the diffusion limit, in which the polarizer behaves as a standard depolarized Raman amplifier, and in the Manakov limit, in which the Raman polarizer behaves ideally, achieving full polarization of the incoming signal in complete alignment to the pump's SOP.

\section{Model and main results}

We consider the simultaneous propagation of two beams in a few kilometers long span of a randomly birefringent telecom fiber. Figure 1 depicts the usual configuration for a co-propagating Raman polarizer, characterized by a low-PMD fiber, a polarized high-power Raman pump and a (typically) randomly polarized input signal at the corresponding first-Stokes wavelength. The main feature which differentiates our theory from most previous studies on fiber-optic Raman amplifiers is its vectorial nature. The first vectorial theory of the Raman effect in randomly birefringent optical fibers was developed by Lin and Agrawal in Ref. [14], and it was applied to describing the operation of what we call here "standard Raman amplifiers". The interest there was on the study of PMD-induced fluctuations in the intensity of the amplified signal.

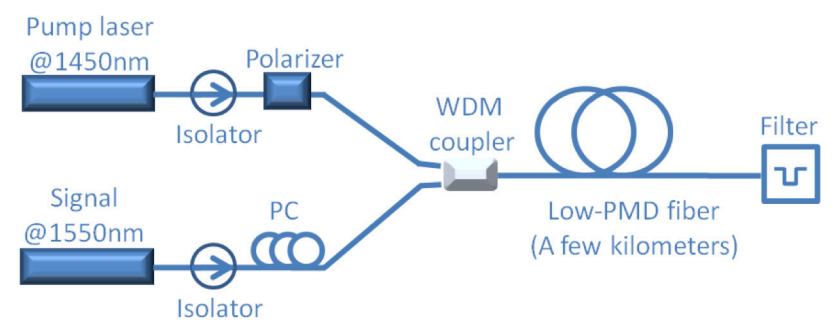

Fig. 1. Schematic depiction of a typical experimental setup with a co-propagating Raman polarizer. Raman pump at $1450 \mathrm{~nm}$ is highly polarized, whereas the state of polarization of the signal is managed with a polarization controller (PC). A wavelength-division multiplexer is used to combine signal and pump and the repolarized signal is demultiplexed at the output with a filter.

Here, on the other hand, we are interested in studying a totally different regime of Raman

\#176299 - \$15.00 USD Received 14 Sep 2012; revised 17 Oct 2012; accepted 17 Oct 2012; published 19 Nov 2012

(C) 2012 OSA

19 November 2012 / Vol. 20, No. 24 / OPTICS EXPRESS 27243 
amplifiers, i.e., that of Raman polarizers. This can be achieved through adequate formulation of the problem in terms of a set of deterministic differential equations. Skipping, for the sake of both focus and space limitations, details of their derivation (which can be found in its entirety in Refs. [2,3]), we can arrive to the equations of motion for the signal Stokes vector:

$$
\partial_{z} \mathbf{S}^{(s)}=\gamma \mathbf{S}^{(s)} \times J_{s}(z) \mathbf{S}^{(s)}+\gamma \mathbf{S}^{(s)} \times J_{x}(z) \mathbf{S}^{(p)}+(g / 2)\left[S_{0}^{(p)} \mathbf{S}^{(s)}+S_{0}^{(s)} J_{R}(z) \mathbf{S}^{(p)}\right] .
$$

where $\gamma$ is the fiber nonlinear coefficient, $J_{S}$ is the self-polarization modulation (SpolM) tensor, $J_{x}-$ cross-polarization (XPolM) modulation tensor and $J_{R}-$ the Raman tensor. All of these tensors are diagonal. The elements of these tensors are dependent on the magnitude of the birefringence beat lengths $L_{B}\left(\omega_{s}\right), L_{B}\left(\omega_{p}\right)$ and on the correlation length $L_{c}$ of the random birefringence variations. The Raman tensor defines the polarization-sensitive amplification of the amplifier: when the elements of this tensor vanish, the model is reduced to a scalar one. Conversely, when the diagonal elements of the Raman tensor keep appreciable values, the theory retains its vectorial character.

Clearly the evolution of the signal Stokes vector sensitively depends on how the elements of these tensors evolve with distance. In order to find their dynamics it is necessary to solve the set of linear ordinary differential equations whose complete derivation and numerical solution is given in Refs. [2,3]. Here we will be concerned only with their approximate analytical solutions, which, as it will be shown, are successfully applicable in the cases of highest interest.

Figures 2(a),(b),(c) demonstrate how well these analytical solutions reproduce the full numerical simulations. Figure 2(a) shows that the elements of the SPolM tensor drop down to zero with distance: they already vanish within the first $10 \mathrm{~m}$ of fiber for most practically relevant situations. Indeed, provided that the length of Raman amplifiers exceeds $1 \div 2 \mathrm{~km}$, we can safely set $J_{s}=\operatorname{diag}(0,0,0)$. Similarly, the elements of the other two tensors also decrease with distance, however at much slower rate. Namely,

$$
\begin{gathered}
J_{x}=-\frac{8}{9} \operatorname{diag}(1,1,1) \exp \left(-z / L_{d}\right) \\
J_{R}=\operatorname{diag}(1,1,1) \exp \left(-z / L_{d}\right)
\end{gathered}
$$

As demonstrated in Fig. 2(b),(c), the decay distance is indeed associated with a characteristic length $L_{d}$, which is called the PMD diffusion length:

$$
L_{d}^{-1}=\frac{1}{3}\left(D_{p} \Delta \omega\right)^{2}
$$

where $D_{p}=2 \sqrt{2} \pi \sqrt{L_{c}} /\left(L_{B} \omega_{p}\right)$ is the PMD coefficient, and $\Delta \omega=\omega_{p}-\omega_{s}$ is the Raman shift $\Delta \omega_{R}=13.2 \mathrm{THz}$.

Our analytical theory can be considered valid only in two limits - the so-called Manakov limit $\left(L_{N L}, L_{R} \ll L_{d}\right)$ and the diffusion limit $\left(L_{N L}, L_{R} \gg L_{d}\right)$ (where $L_{N L}=\left(\gamma S_{0}^{(p)}\right)^{-1}$ is the nonlinear length, and $L_{R}$ is the characteristic amplification length). Please note that the Manakov limit implies the largest values of the Raman matrix elements: therefore the strongest re-polarization occurs in this case. On the other hand, in the diffusion limit all elements of the Raman matrix go to zero, hence no re-polarization occurs.

In the diffusion limit our approximation reduces to the scalar model which describes standard Raman amplifiers, whereas in the Manakov limit we deal with a vectorial Raman amplifier -i.e.

\#176299 - \$15.00 USD Received 14 Sep 2012; revised 17 Oct 2012; accepted 17 Oct 2012; published 19 Nov 2012

(C) 2012 OSA

19 November 2012 / Vol. 20, No. 24 / OPTICS EXPRESS 27244 
a Raman polarizer. Moreover, in the Manakov limit the SPolM, XPolM, and Raman tensors are no longer $z$-dependent, hence the model equations can be solved analytically. This is precisely the situation when we have an ideal Raman polarizer, since the device shown in Fig. 1 performs its signal re-polarization action in the most effective manner possible.
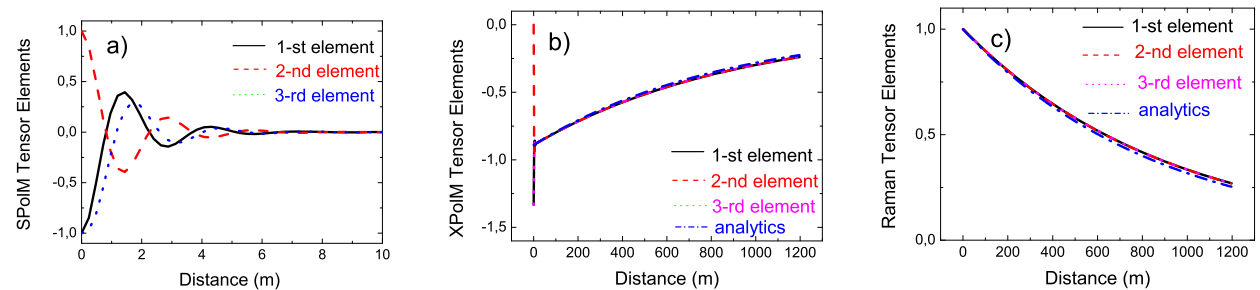

Fig. 2. (a) SPolM, (b) XPolM, and (c) Raman tensors. In Figs. (b),(c) all three curves visually coincide; the blue curve is the analytical result showing exponential decay: $\propto \exp \left(-z / L_{d}\right)$. Parameters are: $L_{c}=1 \mathrm{~m}, L_{B}\left(\omega_{s}\right)=20 \mathrm{~m}, \omega_{p}-\omega_{s}=13.2 \mathrm{THz}, \lambda_{s}=$ $1.55 \mu \mathrm{m}$, and $\lambda_{p}=1.45 \mu \mathrm{m}, L_{d}=870 \mathrm{~m}, \mathrm{PMD}=0.14 \mathrm{ps} \mathrm{km}^{-1 / 2}$.

Let us consider the statistical properties of a Raman polarizer in the undepleted pump regime, by supposing that the signal is initially unpolarized. The immediate questions are - what is the SOP of the outcoming signal beam and how well this beam is polarized? We find that the signal SOP at the fiber output is aligned with the pump SOP, and that the degree of alignment is characterized by the degree of polarization (DOP). The Raman polarizer perfoms its function properly when the output signal DOP stays close to unity. By introducing the gain as $G \equiv$ $\left\langle S_{0}^{(s)}(L)\right\rangle / S_{0}^{(s)}(0)$ we get

$$
G=\frac{1}{2}[1+\exp (g P L)]
$$

and for the DOP:

$$
\mathrm{DOP}=1-G^{-1}
$$

where $P$ is the pump power. Please note that the angular brackets stand for averaging over the ensemble of SOPs of the signal beam. Such ensemble uniformly covers the Poincare sphere at $z=0$. The quantity which characterizes the degree of alignment between the pump and the signal SOPs in a Raman polarizer is the alignment parameter $A_{\uparrow \uparrow}$, which is defined as the cosine of the angle between the output signal SOP and the output pump SOP. We find that $A_{\uparrow \uparrow}=1-G^{-1}$. Please note that although both the DOP and the $A_{\uparrow \uparrow}$ are defined by the same analytical expression in the case of an ideal polarizer, they do not stand for the same physical content. Indeed, the DOP defines the spread of the spot on the Poincare sphere, while the alignment parameter $A_{\uparrow \uparrow}$ defines how close the signal SOP stands to the pump SOP. One could imagine a situation where the tiny spread describing a DOP close to unity is not concentrated near the pump SOP, in which case the DOP and $A_{\uparrow \uparrow}$ are distinctly different. However, this is not our case.

It is also particularly interesting to be able to obtain analytically the polarization-dependent gain (PDG) of the device. A strong PDG is inherent to the operation of a Raman polarizer, and different signal beam SOPs experience different Raman gains: a signal beam with a SOP parallel to the pump Stokes vector is amplified most efficiently, while the orthogonal polarization 
experiences no gain. Indeed, $G_{\max }=\exp (g P L)$ (when the initial SOPs of the pump and signal are parallel) and $G_{\min }=1$ (when the initial pump and signal SOPs are orthogonal). We introduce the PDG parameter $\Delta$ as $\Delta=G_{\max }-G_{\min }$, and obtain that for the ideal Raman polarizer $\Delta=2(G-1)$. A high PDG, though, brings, along with a strong re-polarization of the signal beam, the much less desirable feature of a high level of unwanted relative intensity noise (RIN) generation. Indeed RIN is an important source of potential performance impairment with a Raman polarizer: different methods $[10,11,13]$ have been proposed to combat this problem. The variance of this RIN can be estimated as

$$
\sigma_{s}^{2}=\left\langle S_{0}^{2}(L)\right\rangle /\left\langle S_{0}(L)\right\rangle^{2}-1=\left(1-G^{-1}\right)^{2} / 3
$$

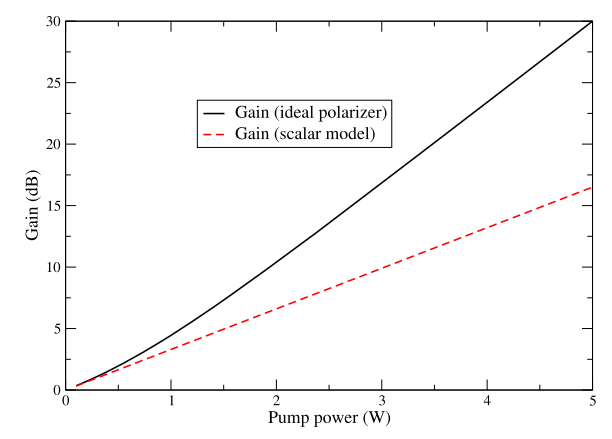

Fig. 3. Gain (in dB) vs. Pump power for the cases of an ideal Raman polarizer (black, straight line) and a standard depolarized Raman amplifier (red, dashed line), with a fixed fiber length of $2 \mathrm{~km}$, and a typical Raman gain coefficient $g=0.76 \mathrm{~W}^{1} \mathrm{~km}^{-1}$ for parallel polarization.

An additional quantity which can be easily derived from our analytical model is the mean gain of an ideal Raman polarizer. It is well known that the gain of a standard Raman amplifier is equal to $g / 2$. In terms of available gain, an ideal Raman polarizer performs much better: from the model we can see that for large values of $G: G \approx \exp (g P L-\ln 2)$, so that the gain coefficient is almost twice that of standard amplifiers. Such property makes Raman polarizers very efficient Raman amplifiers as well. This is illustrated in Fig. 3, where the gain of a standard depolarized amplifier is compared to the gain of an ideal polarizer, for a fixed $2 \mathrm{~km}$ length of standard single-mode fibre, and a variable pump power ranging from 0.1 to $5 \mathrm{~W}$. As expected, in the case of an ideal polarizer with a highly polarized pump, the degree of polarization of the signal and its alignment with the pump grow steadily larger with pump power, and so does the relative gain improvement. At $5 \mathrm{~W}$ pump power, the mean gain for the ideal polarizer is 13.5 $\mathrm{dB}$ higher than that of a standard Raman amplifier.

\section{Counter-propagating configuration}

So far, we have been dealing only with the co-propagating geometry of Fig. 1. In this case, the pump SOP stochastically changes along the fiber, and its output SOP depends on the particular realization of the birefringence stochasticity in the chosen fiber span. Moreover, the stochasticity changes with time, as a result of variations of the environmental conditions. Therefore the trapping of signal's SOP to the pump's SOP does not guarantee the absence of fluctuations of signal's SOP at the output, even though these fluctuations closely follow the time-varying pump SOP. Hence, it is of interest to study the implementation of counter-propagating geometries, which can offer the much desirable stabilization in a laboratory frame (see Refs. [3, 8]). Since the signal's SOP is attracted towards the instantaneous position of pump's Stokes vector,

\#176299 - \$15.00 USD Received 14 Sep 2012; revised 17 Oct 2012; accepted 17 Oct 2012; published 19 Nov 2012

(C) 2012 OSA

19 November 2012 / Vol. 20, No. 24 / OPTICS EXPRESS 27246 
this alignment holds also at the output end of the fiber. The output pump SOP is defined solely by the source, and as such it is supposed to be well defined and deterministic. As regarding the theory, one can repeat the derivations with the opposite sign of the $z$-derivative in the equation governing the evolution of the pump beam. As shown in Ref. [3], this reversing of the sign brings some changes in the components of the XPolM and Raman tensors. They become

$$
\begin{aligned}
& J_{x}^{\text {counter }}=-\frac{8}{9} \operatorname{diag}(1,-1,1) \exp \left(-z / L_{d}\right), \\
& J_{R}^{\text {counter }}=\frac{1}{3} \operatorname{diag}(1,-1,1) \exp \left(-z / L_{d}\right) .
\end{aligned}
$$

The factor $\frac{1}{3}$ in front of the Raman tensor immediately leads to the conclusion that the counter-propagating Raman polarizer is significantly less effective in re-polarization than its co-propagating analog. In order to get similar performances we need either to increase the pump power or lengthen the fiber, or both. If we now solve the equation of motion in the undepleted pump regime, the average gain turns out to be

$$
G=\frac{1}{2}\left(\mathrm{e}^{\frac{2}{3} g P L}+\mathrm{e}^{\frac{1}{3} g P L}\right),
$$

which is significantly smaller than that of a Raman polarizer operating in the co-propagating configuration, although it is still larger than that of an ideal Raman amplifier. For the same value of the product $P L$, the output signal DOP in the conter-propagating configuration is also smaller than in the co-propagating case:

$$
D O P=1-2\left(\mathrm{e}^{\frac{1}{3} g P L}+1\right)^{-1} \approx 1-2 \mathrm{e}^{-\frac{1}{3} g P L}(\text { forgPL } \gg 1) \approx 1-\sqrt{2} G^{-1 / 2} .
$$

As an example, for $G=20 \mathrm{~dB}$ in the co-propagating case the DOP is as high as $99 \%$, while in the counter-propagating configuration it is only $86 \%$. The alignment parameter for the counterpropagating geometry is different from the co-propagating case. Still, for an unpolarized signal, the alignment parameter coincides with the DOP, $A_{\uparrow \downarrow} \approx 1-\sqrt{2} G^{-1 / 2}$.

The PDG parameter $\Delta=G_{\max }-G_{\min }$ is easily calculated, resulting in

$$
\Delta=\frac{1}{2}\left(\mathrm{e}^{\frac{2}{3} g P L}-\mathrm{e}^{\frac{1}{3} g P L}\right)=\frac{1}{2}(1+2 G-\sqrt{1+8 G}) .
$$

Similarly, the RIN is expected to be lower. This is confirmed by its obtained variance:

$$
\sigma_{s}^{2}=\frac{1}{3}\left[1-2\left(\mathrm{e}^{\frac{1}{3} g P L}+1\right)^{-1}\right]^{2} .
$$

In conclusion, we have presented a tractable analytical model for Raman polarizers that is able to predict their most relevant parameters, providing accurate estimations for the output DOP, alignment parameter, PDG and mean gain, as well as being capable of predicting the variance of the RIN noise produced by PDG. This analytical approximation is reduced, in the diffusion limit, to the traditional description of the depolarized Raman amplifier, whereas in the Manakov limit it describes the behavior of an ideal Raman polarizer.

\section{Acknowledgments}

The authors wish to thank the financial support of Italian Ministry of University and Research project no.2008MPSSNX and the Spanish Ministerio de Economía y competividad (MINECO) grant TEC2011-27314. The work of Javier Nuño is partly supported by the Ministerio de Economía y competividad (MINECO) through an FPI Fellowship.

\#176299 - \$15.00 USD Received 14 Sep 2012; revised 17 Oct 2012; accepted 17 Oct 2012; published 19 Nov 2012

(C) 2012 OSA

19 November 2012 / Vol. 20, No. 24 / OPTICS EXPRESS 27247 